\title{
Avaliação perioperatória da viabilidade intestinal ${ }^{\star}$
}

\author{
Perioperative assessment of the intestinal \\ viability
}

\author{
Fernando Pinho Esteves ${ }^{(1)}$, Pedro Paulo de Paris Caravatto(2), \\ Lucas Yugo Shiguehara Yamakami( ${ }^{(3)}$, Sérgio Eduardo Alonso \\ Araújo( $^{(4)}$, Tércio Genzini(4), Marcelo Perosa(4)
}

\begin{abstract}
Esteves FP, Caravatto PPP, Yamakami LYS, Araújo SEA, Genzini T, Perosa M. Avaliação perioperatória da viabilidade intestinal. Rev Med (São Paulo) 2003 jan.-dez.;82(1-4):46-57.

RESUMO: A determinação da viabilidade de segmentos intestinais e do grau de isquemia a partir de critérios clínicos pode ser de extrema dificuldade para o cirurgião. Faz-se necessário lançar mão de métodos auxiliares para se atestar o grau de comprometimento da alça intestinal, muitas vezes inaparente à avaliação clínica. $\mathrm{O}$ presente trabalho apresenta uma revisão de literatura dos principais métodos existentes para se fazer essa avaliação, descrevendo a técnica e equipamentos necessários para cada um, bem como os resultados da aplicação dos mesmos. Os principais métodos auxiliares encontrados na literatura se dividem em experimentais: eletromiografia, laser Doppler sem contato e Imagem térmica; e clínicos: avaliação exclusiva por critérios clínicos, Doppler ultrassom, laser Doppler, injeção intravascular de corantes, oximetria superficial, oximetria de pulso, termometria, pHmetria, tonometria e fotopletismografia infravermelha. São também reinterados os resultados da indicação e realização das relaparotomias no manejo de pacientes com insuficiência vascular mesentérica. Após a revisão de literatura, pudemos concluir que o Doppler ultrassom e a injeção intravenosa de fluoresceína representam os métodos auxiliares para a avaliação perioperatória da viabilidade intestinal mais utilizados devido principalmente ao seu baixo custo e relativa facilidade de uso. Optamos por destacar o papel da laparoscopia pós-operatória no segmento de pacientes submetidos ao tratamento cirúrgico de insuficiência vascular mesentérica, em especial naqueles em que a avaliação da viabilidade intestinal foi duvidosa.
\end{abstract}

DESCRITORES: Assistência perioperatória. Procedimentos cirúrgicos do sistema digestivo/métodos. Isquemia/complicações. Intestinos/patologia. Intestinos/irrigação sangüínea. Circulação explânemica. Literatura de revisão.

\footnotetext{
* Prêmio Monografias - XX COMU 2001.

(1) Acadêmico do $3^{\circ}$ ano de Medicina da Faculdade de Ciências Médicas da Santa Casa de São Paulo - FCMSCSP.

(2) Acadêmico do $4^{\circ}$ ano de Medicina da Faculdade de Ciências Médicas da Santa Casa de São Paulo - FCMSCSP

(3) Acadêmico do $4^{\circ}$ ano de Medicina da Faculdade de Medicina da Universidade de São Paulo - FMUSP.

(4) Orientador, Mestre em Cirurgia pela Faculdade de Medicina da Universidade de São Paulo - FMUSP.

Endereço para correspondência: Fernando Pinho Esteves. Rua Dr. Heitor Pereira Carrilho, 11 - Freguesia do Ó - São Paulo, SP. CEP: 02729-000. e-mail: fernandopipoca@ hotmail.com
} 


\section{MÉTODOS DE AVALIAÇÃO DA VIABILIDADE INTESTINAL: revisão de literatura}

\section{INTRODUÇÃO}

A avaliação peri-operatória de segmentos intestinais possivelmente isquêmicos apresenta-se sempre como tarefa de extrema dificuldade para o cirurgião ${ }^{71}$, persistindo controvérsias acerca do melhor método ${ }^{80}$.

A determinação e ressecção precisas da porção comprometida do intestino são fundamentais para o sucesso do procedimento, bem como para a exclusão de

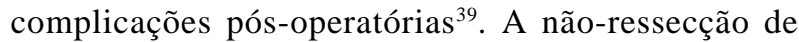
porções inviáveis do intestino leva geralmente à deiscência da anastomose e sepsis, da mesma forma que a permanência de intestino ainda viável, porém já com lesões irreversíveis em sua parede, pode fazer com que o paciente evolua com complicações como a colite ou a estenose intestinal.

A ressecção desnecessária de grande extensão de intestino ainda viável pode, por outro lado, levar à outras complicações como a chamada Síndrome do Intestino Curto, implicando em significativa redução da capacidade absortiva do paciente e até na necessidade de nutrição parenteral. A preservação de alguns centímetros de intestino pode determinar a diferença entre um paciente capaz de absorver uma dieta oral adequada e um paciente dependente de nutrição parenteral ou até mesmo de transplante intestinal ${ }^{39,71}$.

Em face das inúmeras complicações oriundas da ressecção de segmentos intestinais viáveis e da permanência de segmentos intestinais inviáveis na cavidade, faz-se necessário o uso de métodos acurados para a avaliação intra-operatória da viabilidade intestinal.

A presente revisão tem por objetivo analisar os resultados da aplicação dos principais métodos disponíveis para a avaliação intra-operatória da viabilidade intestinal. Foram ainda revisadas a indicação e os resultados da realização de relaparotomias programadas no manejo de doentes com suspeita ou diagnóstico de insuficiência vascular do intestino. Os métodos foram classificados de acordo com a fase de aplicação, estando divididos em experimentais e clínicos. Foi considerado clínico todo o método eu apresentasse pelo menos um relato de sua aplicação em pacientes.

\section{MÉTODOS EXPERIMENTAIS}

São classificados como métodos experimentais aqueles cuja aplicação não se encontra relatada em séries clínicas, representando, portanto, uma futura opção para a avaliação da viabilidade intestinal perioperatória em pacientes.

\section{Eletromiografia intestinal}

A eletromiografia determina a viabilidade intestinal através da avaliação da atividade das ondas lentas na parede do intestino. O estado isquêmico gera alterações previsíveis nos padrões de atividade dessas ondas lentas, sendo que a sua freqüência diminue progressivamente com o aumento do tempo de isquemia quente ${ }^{34,44}$. Um estudo recente de Lammers et al. ${ }^{47}$ mostrou em gatos que na isquemia mesentérica aguda há uma diminuição progressiva e não-homogênea na condução das ondas lentas e na excitabilidade local da parede intestinal.

Outros estudos ${ }^{17,21}$ tentaram relacionar a motilidade e o fluxo sangüíneo intestinal e mostraram que segmentos intestinais submetidos a isquemia quente por até 18 horas sobreviviam quando revascularizados, apresentando, porém, contratilidade diminuída de suas paredes. Essa diminuição era oriunda da destruição de boa porção das células musculares lisas e ganglionares pelo estado isquêmico.

Visando correlacionar a condutância elétrica e a isquemia tecidual, Brolin et al. ${ }^{11,13}$ criaram um aparelho capaz de quantificar o grau de dano isquêmico da parede intestinal através avaliação da atividade miocontrátil dessa parede. Este aparelho (medidor de contratilidade elétrica) consiste em um duplo clamp de metal que possui um sensor para detecção de variações tanto longitudinais quanto transversais da parede intestinal (Figura 1). Este conjunto é conectado ainda a uma fonte de estímulo por corrente constante (que é descarregada por eletrodos na ponta dos clamps) e a um eletrocardiógrafo para registro gráfico da atividade elétrica da parede.

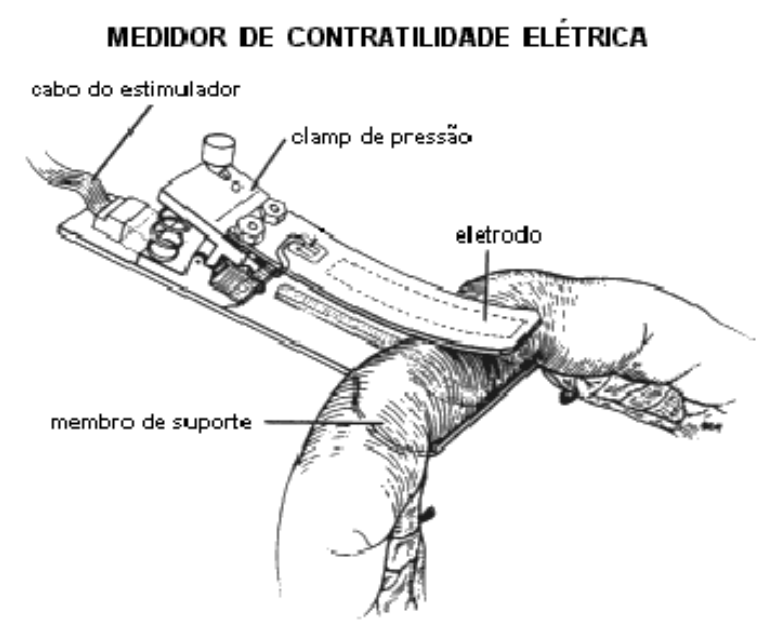

Figura 1. Medidor de contratilidade elétrica. (Reproduzido de: Brolin RE, Semmlow JL, Koch RA, et al. Myoeletric assessment of bowel viability. Surgery, 102(1):32-8, 1987, Fig. 3, p.35).

Para a operação do medidor de contratilidade elétrica, este deve ser conectado à serosa do intestino e, a partir daí o eletrocardiógrafo já pode passar a registrar a atividade elétrica intestinal. Com relação à fonte de estímulo, a partir do comando do operador, produzir 
descargas elétricas sucessivas que após 10 a 15 segundos já começam a desencadear respostas contráteis

Usando o presente aparelho, esse mesmo grupo ${ }^{12}$ encontrou, em um estudo experimental, significativa correlação entre a quantidade de corrente necessária para produzir uma contração muscular bem definida e o grau de lesão em que se encontrava o segmento intestinal, tanto na avaliação clínica quanto com Doppler ultrassom. Dessa forma, quanto maior fosse a deterioração na cor e a extensão do desaparecimento dos sinais de doppler na parede intestinal, arteríolas periféricas e artérias marginais, uma carga cada vez maior de miliamperes seria necessária para produzir uma contração visível no intestino. Segundo esse estudo, os segmentos intestinais que respondessem à estímulos de 30 a $50 \mathrm{mAmp}$ poderiam se encontrar visualmente isquêmicos, porém ainda estariam viáveis.

Segundo Semmlow et al. ${ }^{76}$, por ser passível de melhor quantificação, a análise mioelétrica do intestino pode ser um parâmetro mais confiável para a avaliação da viabilidade intestinal do que os testes baseados na avaliação do fluxo sanguíneo e da aparência do intestino.

Em um estudo experimental com um modelo de isquemia por obstrução arterial, Orland et al.$^{61}$ relataram diminuição estatisticamente significante da atividade elétrica intestinal nas alças inviáveis, significância esta que não foi observada com relação ao aumento do estímulo necessário para a produção de contração muscular nos crescentes graus de isquemia tecidual; esses resultados foram também observados por um estudo de Brolin et al.. .

Uma desvantagem da eletromiografia reside no efeito que a avaliação subjetiva pode ter na interpretação dos resultados aferidos com as descargas elétricas nas alças possivelmente isquêmicas. Com o intuito de anular essa influência, autores ${ }^{10,14}$ vêm se engajando na criação de um medidor de contratilidade elétrica modificado, este contendo uma espécie de microprocessador que monitore os estímulos e quantifique via computador as respostas gráficas obtidas, eliminando assim a necessidade de avaliação visual, muitas vezes equivocada, da contração muscular de resposta ao estímulo. Em recente estudo com a introdução de um medidor de contratilidade elétrica modificado, Basdanis et al..$^{5}$ relataram que, a diminuição dos erros de subjetividade alcançada com o uso desse medidor produziu resultados mais confiáveis e realistas, logo justificando seu estudo em séries clínicas.

\section{Laser Doppler sem contato}

O presente método tem o mesmo princípio do laser Doppler, diferenciando-se desse pelo fato de o aparelho apresentar inovações tecnológicas em seus circuitos que eliminam os efeitos da luz refletida pela parede intestinal e de alterações temporais (entre o transdutor e a parede intestinal) que possam influenciar no resultado obtido. Essas inovações refletem na nãonecessidade do contato entre o probe e a parede intestinal, anulando assim a influencia do peristaltismo e da pressão e ângulo do probe em relação à essa parede, fatores que podem diminuir a precisão do método e aos quais estão suscetíveis o laser Doppler e Doppler ultrassom.

Estudando o presente método, Ando et al. ${ }^{3}$ demonstraram em um estudo experimental com modelo de isquemia por obstrução arterial uma taxa de resultados falso-positivos de $12 \%$.

\section{Imagem Térmica (IT)}

Este método consiste na detecção, por meio de uma câmera especial apontada para o campo operatório, da radiação infravermelha emitida pela alça intestinal. As imagens obtidas são projetadas em um monitor sendo obtida uma coloração branca para as porções quentes e vascularizadas e uma coloração negra para as porções fria e isquêmicas. A câmera é sensível para diferenças de temperatura até menores que $0,1^{\circ} \mathrm{C}$, sendo hipoteticamente isquêmica a porção da alça que apresentar diminuição na emissão de radiação infravermelha.

Em um estudo com porcos, Brooks et al. ${ }^{15}$ demonstraram, utilizando o método em um modelo de isquemia mista, a completa ausência de resultados falsopositivos, sendo a taxa de resultados falso-negativos igual a $30,5 \%$.

\section{MÉTODOS CLÍNICOS}

São classificados como métodos clínicos aqueles cuja aplicação já foi relatada em séries clínicas.

\section{Avaliação por critérios clínicos}

Classicamente, a avaliação da viabilidade intestinal apenas por critérios clínicos começa com a inspeção das alças. Em seguida, com exceção dos casos de necrose avançada, o cirurgião realiza a revascularização do intestino com a eliminação da causa da isquemia (estrangulamento, trombo, etc), prosseguindo com lavagem peritoneal da cavidade abdominal com solução salina aquecida ${ }^{18}$ ou envolvendo as alças em soro morno por um período de 10 a 15 minutos $^{39}$. As manobras de aquecimento do intestino visam reduzir o espasmo vascular e aumentar a circulação no intestino como um todo, permitindo assim uma melhor avaliação clínica da viabilidade intestinal ${ }^{39}$. A concomitante injeção intraarterial de papaverina também pode ser realizada uma vez que esta também atua diminuindo o espasmo arterial ${ }^{71}$.

Os parâmetros utilizados para a avaliação da viabilidade são a cor da alça, que varia com a duração da isquemia e com o tipo de obstrução vascular (arterial, venosa ou mista), a presença de peristaltismo, a presença de pulso arterial e o sangramento à secção da parede intestinal.

A alça intestinal que apresente coloração rósea é invariavelmente viável, enquanto que alças com coloração 
enegrecida, apresentando paredes flácidas, finas, distendidas e com conteúdo sanguinolento quase sempre sugerem necrose (Figura 2). As oclusões venosas resultam em congestão intestinal e mesentérica, com grande enegrecimento da alça, ao passo que oclusões arteriais pouco influem na aparência do intestino. Nos casos em que a alça intestinal apresentar alternância entre regiões róseas e escuras deve-se sempre pensar em isquemias nãooclusivas que resultam em baixo fluxo sangüíneo para a alça $\mathrm{a}^{39,61,71}$.

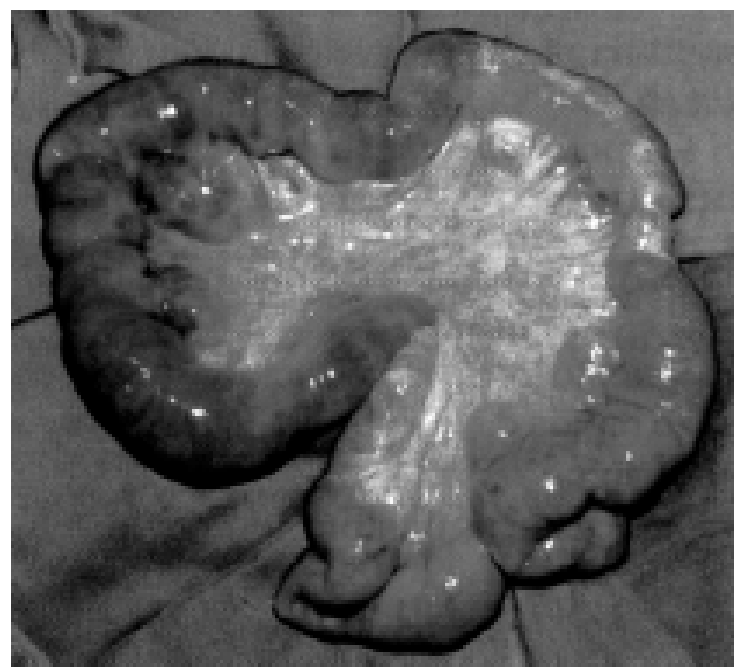

Figura 2. Aspecto macroscópico do intestino necrosado. (Retirado de: Savassi Rocha PR. Avaliação peroperatória da viabilidade intestinal. In: Rasslan S. O doente cirúrgico na UTI. CBMI - Série Clínicas Brasileiras de Medicina Intensiva. v.10, p.412-29, 2001. Fig. 23.3, p. 412).

Com relação à atividade peristáltica, esta cessa nos estados isquêmicos, retornando quando há revascularização de alças ainda viáveis. Apesar disso, está comprovado que o peristaltismo não é uma medida segura da viabilidade intestinal ${ }^{37}$, uma vez que em comprometimentos vasculares irreversíveis a atividade peristáltica se mantém sob a forma de espasmo anóxico, sendo esse facilmente confundido com peristaltismo normal $^{39,61,71}$.

A avaliação da presença de pulso arterial também não é bom método para a avaliação da viabilidade, uma vez que em isquemias de origem venosa o pulso arterial costuma desaparecer tardiamente e após o desenvolvimento de infarto franco. Além disso as pulsações podem ser observadas após a revascularização intestinal, mesmo quando a necrose irreversível já se instalou na alça ${ }^{39}$.

O sangramento após secção da alça é um bom indicador nas isquemias intestinais de origem arterial, mas pode ser um dado falho nas obstruções venosas, uma vez essas situações apresentam sangramento maior que o habitual $^{71}$. No momento de uma possível ressecção intestinal, esta deve proceder até se encontrar a mucosa com aspecto normal e apresentando bom sangramento ${ }^{71}$. Apesar disso, podem existir segmentos ainda viáveis que não apresentem sangramento nas bordas ou que sangrem em excesso (isquemias venosas) ${ }^{39}$.

As isquemias de intestino grosso requerem manejo e cuidado mais delicado por parte do cirurgião pois, além de possuir atividade peristáltica mais escassa, este órgão apresenta alterações de coloração mais tardias quando em estado isquêmico ${ }^{71}$.

Bulkeley et al. ${ }^{16}$ demonstraram em seus estudos que a avaliação da viabilidade intestinal apenas por critérios clínicos apresenta taxa de falso-positivo de $36 \%$ e falsonegativo de $46 \%$. Savassi-Rocha et al. ${ }^{73}$ demonstraram que a avaliação apenas por critérios clínicos apresenta falhas na detecção da viabilidade em até $50 \%$ dos casos com lesão intestinal intermediária.

\section{Doppler Ultrassom (DU)}

O DU é um aparelho capaz de detectar, por meio da incidência de um feixe de ultra-som de baixa frequiência ( 5 a $10 \mathrm{MHz})^{71}$, o fluxo sangüíneo em vasos tão pequenos quanto artérias digitais sendo, portanto, largamente utilizado no diagnóstico de doenças vasculares periféricas ${ }^{39}$. A frequiência do som é alterada quando este é refletido por estruturas em movimento (hemácias por exemplo), condicinando a detecção do fluxo sangüíneo ${ }^{68}$.

A técnica para operação do mesmo é muito simples e consiste na aplicação do transdutor estéril do DU à margem anti-mesentérica do intestino (pois possui vascularização mais precária), incidindo com o mesmo em um ângulo de $45^{\circ}$ com a parede intestinal e untando a extremidade do sensor com algum tipo de gel para aumentar a sensibilidade (Figura 3). Não se deve exercer muita pressão sobre a parede intestinal para não dificultar o fluxo em pequenos vasos $22,39,71$. A presença de ruídos arteriais em isquemias arteriais, mistas ou de baixo fluxo pressupõe viabilidade do segmento intestinal em questão ${ }^{71}$. O uso do audiofone pode facilitar a técnica, aumentando a acuidade do método ${ }^{75}$, mas o registro gráfico não incorre em nenhum melhoramento ${ }^{16}$. A ausculta de alças adjacentes à alça isquêmica é imprescindível para a exclusão de fatores sistêmicos (como hipotensão) que possam levar à uma ausculta falso-negativa ${ }^{71}$.

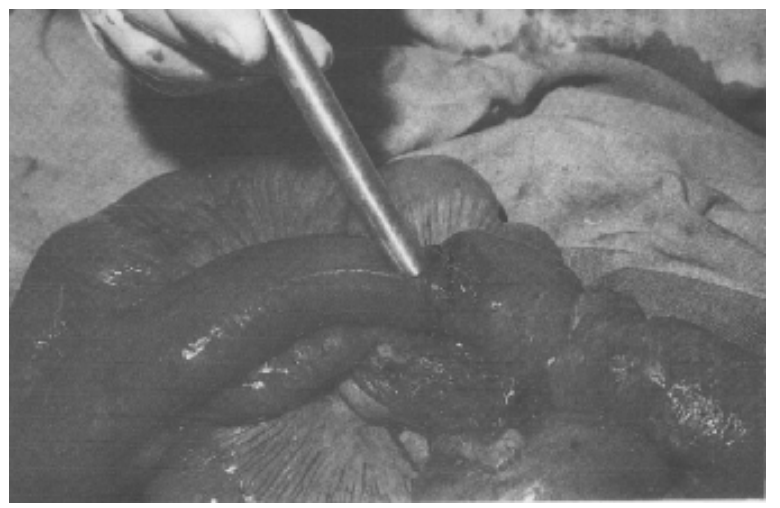

Figura 3. Aplicação do Doppler ultrassom. (Reproduzido de: Savassi Rocha PR. Métodos de avaliação da viabilidade intestinal. In: Rasslan S. Afecções cirúrgicas de urgência. São Paulo: Robe; 1995. p.186, Fig. 2). 
Wright e Hobson ${ }^{95}$ foram os primeiros a estudar essa técnica e demonstraram que a presença de pulso em alça na avaliação intraoperatória determinava a viabilidade do segmento intestinal analisado, esta sendo comprovada em laparotomias realizadas 24 a 48 horas após o fechamento da cavidade.

Em estudos experimentais, Cooperman et al. ${ }^{24}$ demonstraram ser viável a anastomose da alça intestinal em até $1 \mathrm{~cm}$ de distância do último sinal de Doppler audível. Em duas séries clínicas (117 e 23 pacientes), este mesmo grupo demonstrou com o DU a presença de fluxo arterial em segmentos intestinais considerados clinicamente inviáveis ${ }^{10}$, relatando a inexistência de complicações pósoperatórias em pacientes que permaneceram com alças intestinais dessa natureza em suas cavidades ${ }^{23}$.

A detecção de pulso pelo DU mostrou ser experimentalmente e em um estudo em três pacientes, um bom indicador da viabilidade intestinal em isquemias mesentéricas secundárias à trombose arterial ${ }^{60,95}$. A eficácia na avaliação da viabilidade intestinal em isquemias arteriais se mostrou igual a $86 \%$ em um estudo de Lynch et al. ${ }^{52}$.

A eficácia do DU no diagnóstico da viabilidade em alças estranguladas que foram revascularizadas depende do tempo de estrangulamento. O método se mostra eficaz quando o tempo de estrangulamento for inferior a 2 horas, enquanto que para tempos de estrangulamento maiores os critérios clínicos são mais confiáveis $^{91}$.

O uso do DU também foi preconizado para a avaliação da necessidade de reconstrução da artéria mesentérica inferior após cirurgias de aorta abdominal ${ }^{38}$.

Apesar da larga aceitação do método, um estudo de Bulkley et al. ${ }^{16}$ não encontrou aumento da acurácia com o DU em relação a avaliação da viabilidade intestinal apenas por critérios clínicos, resultados suportados também por Orland et al. ${ }^{61}$.

\section{Laser Doppler (LD)}

Este método consiste em um refinamento do DU, diferenciando-se do último pelo fato de ser baseado na incidência de luz ao invés de ondas sonoras ${ }^{68}$. Uma vez que a penetração do laser no tecido é de $1 \mathrm{~mm}$ e fluxos sanguíneos de até $1 \mathrm{~mm}$ por segundo podem ser detectados, os achados com essa técnica apresentam maior proporcionalidade e precisão em relação à velocidade de circulação do sangue ${ }^{39,68}$. Estudos experimentais ${ }^{31,68,79}$ desenvolvidos inicialmente para o estudo dessa técnica já apontavam para sua maior sensibilidade.

Ahn et al. ${ }^{1}$, no primeiro estudo clínico com o método questionaram até que ponto a medição individual do fluxo na serosa e mucosa do intestino refletiam a perfusão sangüínea total na parede intestinal, uma vez que nos estados isquêmicos a parada da circulação capilar se inicia nas camadas mais internas posteriormente se instalando nas mais externas ${ }^{68}$.

O estudo e uso da técnica continuou largamente relatado $^{28,40,41,50}$, sendo o LD sempre referido como um método de alta precisão na determinação da viabilidade intestinal. Um estudo comparativo entre o LD e o DU mostrou uma sensibilidade do primeiro de $94 \%$ e do segundo de $86 \%{ }^{52}$.

Em isquemias arteriais, a sensibilidade, precisão e o valor preditivo positivo com essa técnica se mostraram em torno de $100 \%$, sendo o valor preditivo negativo igual a $69 \%$ 66,67,79.

Uma desvantagem do LD é o fato desse método ser mais caro e mais passível de erros de interpretação, devido à sua maior sensibilidade ${ }^{71}$.

\section{Injeção intravascular de corantes}

A injeção intravascular de um corante resulta na sua distribuição pelo organismo e absorção pelos tecidos. Estando o sistema vascular pérvio e o tecido viável, a visualização macroscópica da absorção dessas substâncias especiais deve ser sempre possível, sendo esse o princípio do presente método ${ }^{39,71}$.

Muitos corantes já foram estudados com o fim de avaliar a viabilidade intestinal, destacando-se a fluoresceína, o azul de Evans ${ }^{26}$, o bromofenol azul ${ }^{26}$, o azul patente $\mathrm{V}^{46,58,64}$, o azul de Tripan ${ }^{64}$ e o brometo de tetrazólio ${ }^{19}$. Alguns desses apresentaram relativo sucesso como azul patente $\mathrm{V}$ e o azul de trypan, sendo a eficácia deste último na determinação de porções intestinais inviáveis igual a $84 \%{ }^{64}$ segundo Papachristou et al. ${ }^{64}$. A fluoresceína sempre foi o corante mais largamente estudado e utilizado.

A fluoresceína (resorcinolftaleína) é um corante que, uma vez injetado na circulação, cora os tecidos durante 12 a 30 horas, sendo excretado e eliminado da circulação em cerca de 5 horas após sua injeção. Esse corante tem a propriedade de emitir fluorescência quando incidido com luzes que apresentem maior comprimento de onda, como a luz ultravioleta. A lâmpada da Wood pode ser utilizada para a obtenção dessa luz ${ }^{71}$.

A técnica para aplicação do método consiste na injeção intravenosa da fluoresceína sódica $10 \%$ numa dose de $10 \mathrm{mg} / \mathrm{kg}$ de peso, aguardando-se 3 minutos para a leitura. Após esses 3 minutos deve-se exteriorizar e identificar a alça(s) comprometida(s), incidindo sobre essa(s) a luz da lâmpada de Wood (à uma distância de 30 cm e com a luz ambiente desligada) e avaliando-se a fluorescência emitida ${ }^{71}$ (Figura 4). 

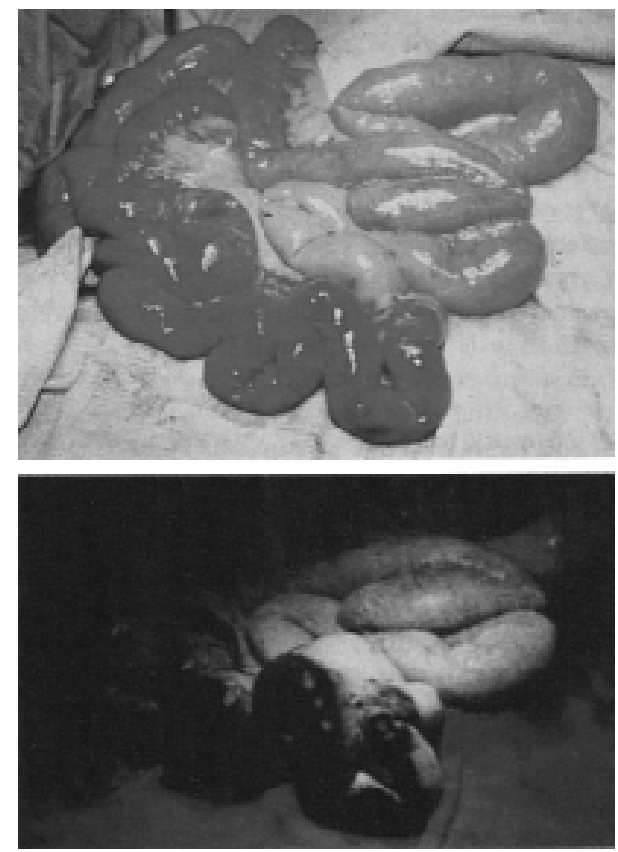

Figura 4. Fotos apresentando o aspecto do intestino após a abertura da cavidade (acima) e sob a luz ultravioleta após a injeção da fluoresceína (abaixo). (Reproduzido de: Horgan PG, Gorey TF. Operative assessment of intestinal viability. Surg Clin North Am 1992; 1:141-53, Fig. 1).

As alças intestinais que emitirem fluorescência homogênea devem ser consideradas viáveis, enquanto que as que não emitirem fluorescência deve ser consideradas inviáveis ${ }^{20}$. Nos casos de fluorescência heterogênea a viabilidade deve ser sempre considerada duvidosa. Para esses casos duvidosos, Stolar e Randolph ${ }^{86}$ desenvolveram a seguinte escala correlacionando três padrões anormais de fluorescência com suas alterações histopatológicas:

$>$ Tipo 1 - distribuição reticular difusa sobre as áreas de fluorescência: $100 \%$ dos segmentos nessas condições sobrevivem;

> Tipo 2 - distribuição irregular da fluoresceína com áreas extensas de não-fluorescência: os segmentos nessas condições se edemaciam quando deixados na cavidade, havendo perda de mucosa, hemorragia e fibrose;

> Tipo 3 - corante retido por 24 horas sendo visto em remendos grandes e confluentes: os segmentos nessas condições necrosam se deixados na cavidade.

A fluoresceína foi inicialmente utilizada por Herrlin et al. ${ }^{37}$ que documentaram em 1942 a avaliação da árvore vascular mesentérica de 500 pacientes. Desde então muitos estudos experimentais ${ }^{28,32,33,42,48,52,65}$ e clínicos ${ }^{16,63}$ têm sido desenvolvidos para a avaliação do uso da fluoresceína na determinação da viabilidade intestinal.

Bulkley et al. ${ }^{16}$ demonstraram em uma série de 28 pacientes a superioridade da fluoresceína frente aos critérios clínicos e ao Doppler ultrassom na determinação da viabilidade intestinal, sendo esses resultados suportados também por alguns estudos experimentais ${ }^{15,52,55}$.

Estudos de Gorey et al..$^{33}$ com o presente método registraram sensibilidade e especificidade de $96 \%$ e $95 \%$ na identificação do intestino inviável, apresentando, contudo, uma taxa de falsos positivos de $17 \%$ nas oclusões venosas.

Segundo Savassi-Rocha et al. ${ }^{75}$ o uso da fluoresceína é o método mais eficaz para a determinação peri-operatória da viabilidade intestinal, sendo, contudo, pouco efetivo na determinação de pontos ótimos de ressecção intestinal, uma vez que não produz uma linha de demarcação nítida entre intestino viável e não-viável.

O principal problema que envolve o uso da fluorescência consiste na influência que a avaliação subjetiva do cirurgião pode ter sobre os resultados alcançados. Partindo desse princípio, alguns autore ${ }^{20,81,82,83}$ introduziram a utilização de um fluorâmetro para a quantificação dos achados após a injeção da fluoresceína. Este aparelho capta e quantifica a fluorescência obtida, que é analisada por um software de computador, que por sua vez produz gráficos indicando os locais viáveis e não-viáveis do intestino analisado. Silverman et al. ${ }^{83}$ registraram um aumento da precisão na determinação da viabilidade intestinal de 53\% com a avaliação subjetiva do cirurgião para $98 \%$ com o uso do fluorâmetro.

O uso da fluoresceína apresenta baixíssimas taxas de complicações, essas ocorrendo em torno de $0,6 \%{ }^{85}$ dos pacientes e constituindo-se principalmente por reações alérgicas. Reações sistêmicas graves tais como insuficiência respiratória, edema facial, choque, dispnéia e broncoespasmo, entre outros, são excepcionais.

\section{Oximetria de superfície}

Baseado no fato de que o estado isquêmico produz uma pronunciada redução na $\mathrm{PO}_{2}$ (pressão parcial de $\mathrm{O}_{2}$ ) tecidual, o presente método avalia a viabilidade intestinal pela correlação entre a tensão superficial de oxigênio na serosa intestinal e o grau de lesão isquêmica do órgão.

A medição da $\mathrm{PO}_{2}$ com esse método se faz pela colocação de um eletrodo na margem anti-mesentérica intestinal, estando este conectado a um oxímetro. A quantificação da oxigenação superficial dos tecidos duvidosos se dá pela comparação desses com o tecido normal.

Em um estudo experimental de Locke et al..$^{51}$, foi evidenciada deiscência e necrotização em todas as alças intestinais que foram anastomosadas apresentando até $30 \%$ do nível basal de oxigenação; ainda nesse estudo $1 / 3$ dos segmentos apresentando entre 30 e $50 \%$ da oxigenação normal necrosaram, não havendo deiscência dos segmentos com mais de $50 \%$ de saturação de oxigênio.

Em um estudo com ratos, Sheridan et al. ${ }^{80}$ conseguiram $92,5 \%$ de precisão na previsão da viabilidade intestinal com o presente método.

\section{Oximetria de pulso}

A oximetria de pulso baseia-se nos mesmos 
princípios da oximetria superficial, diferenciando-se dessa pelo fato de mensurar simultaneamente e perfusão e oxigenação tecidual ${ }^{14,25,53}$.

A técnica para aplicação do método consiste na fixação do probe do oxímetro à borda anti-mesentérica do intestino para o registro dos parâmetros pelo aparelho. Segundo um estudo experimental de Turkyilmaz et al. ${ }^{89}$, uma $\mathrm{SaO}_{2}$ maior que $80 \%$ indica viabilidade, enquanto valores entre $70 \%$ e $80 \%$ (índice de fístula $<10 \%$ ), entre $70 \%$ e $60 \%$ (índice de fístula de $75 \%$ ) e abaixo de $60 \%$ ( $100 \%$ de fístula) indicam respectivamente isquemias leve, moderada e grave.

Os resultados encontrados com a técnica até o presente momento apontam para a sua superioridade frente ao Doppler ultrassom e as critérios clínicos na avaliação da viabilidade intestinal ${ }^{25,53,89}$.

\section{Termometria}

A temperatura dos tecidos é influenciada por seu aporte sanguíneo. Dessa forma, a diminuição do calibre dos vasos intramurais intestinais no seu percurso em direção à margem antimesentérica explica a existência de uma diferença de temperatura entre as margens mesentérica e antimesentérica do intestino ${ }^{92,93}$. Estudos mostram que essa diferença varia de 0,9 a $3,8^{\circ} \mathrm{C}$ no rato $^{75}$, enquanto que no organismo humano ela é de aproximadamente $1,6^{\circ} \mathrm{C}^{72}$.

Quando o intestino entra em isquemia há um desaparecimento progressivo dessa diferença de temperatura, sendo o princípio da termometria o estudo da correlação entre a diminuição dessa diferença e o grau de isquemia do intestino ${ }^{71}$.

Para a aplicação do método deve-se medir a temperatura das margens mesentérica e antimesentérica com a alça estudada envolvida por compressas e com os focos de luz apagados, medidas essas que contribuem para a minimização da influência de fatores externos nos resultados aferidos. Diferenças de temperatura entre essas margens inferiores a $0,5^{\circ} \mathrm{C}$ sugerem necrose da alça ${ }^{75}$.

Deve-se aferir as temperaturas também dos segmentos intestinais adjacentes à alça duvidosa, pois alguns fatores sistêmicos (hipotensão arterial por exemplo) também podem ser responsáveis pelo desaparecimento desse gradiente, limitando nesses casos a utilização do método $^{71}$.

Estudos mostraram que o método é útil nas isquemias arteriais e mistas, demonstrando nesses casos resultados semelhantes ao Doppler ultrassom ${ }^{72,73,75}$. Em contra-partida, um estudo de Diniz MTC mostra que nas isquemias por oclusão venosa o desaparecimento do gradiente térmico pode acontecer com o intestino ainda viável, concluindo portanto que, o desaparecimento do gradiente de temperatura não significa necessariamente necrose intestinal ${ }^{27}$.

O comportamento da termometria nas alças estranguladas e revascularizadas depende do tempo de estrangulamento, sendo eficaz em estrangulamentos abordados com até duas horas de duração ${ }^{91}$.

\section{pHmetria e tonometria de supefície}

A redução do aporte de oxigênio oriunda do estado isquêmico resulta em uma rápida mudança do metabolismo aeróbico para o anaeróbico, com instalação da glicólise anaeróbica e consequente aumento da $\mathrm{pCO}_{2}$ e diminuição do $\mathrm{pH}$ tecidual ${ }^{71}$.

Partindo desse princípio, alguns autores ${ }^{43,57}$ começaram a estudar a relação entre o grau de isquemia intestinal e os resultados obtidos com a pHmetria e a tonometria (medida da $\mathrm{pCO}_{2}$ ) na superfície serosa do intestino. Nesses estudos observou-se que essas alterações de $\mathrm{pH}$ e $\mathrm{pCO}_{2}$ são restabelecidas quando ocorre a revascularização do segmento isquêmico, estando ele viável ou não ${ }^{90}$. Dessa forma, os autores puderam concluir que o $\mathrm{pH}$ e a $\mathrm{PCO}_{2}$ não depende da viabilidade intestinal e sim do aporte sanguiíneo ao tecido.

Uma vez que não existem estudos mostrando a existência de um nível crítico de acidez para o qual o intestino ainda se encontre viável ${ }^{71}$, o presente método se mostra bom apenas para a detecção precoce do estado isquêmico (diminuição do aporte sangüíneo), não sendo eficaz na determinação da viabilidade intestinal ${ }^{39}$.

\section{Fotopletismografia infravermelha}

Essa técnica consiste na monitoração de mudanças no volume sanguíneo tecidual através da detecção de alterações no grau de reflexão da luz infravermelha pela parede intestinal, sendo essas alterações oriundas de variações na absorção de luz pela hemoglobina ${ }^{39,71}$. O controle é estabelecido usando-se a superfície do estômago sadio como referência e considerase necrosado o segmento intestinal que apresente até 50\% da reflexão de luz do controle ${ }^{39}$.

Em estudos com cachorros, Pearce et al. ${ }^{65}$ relataram a eficácia do método de $100 \%$, contra $88 \%$ do DU e do clareamento com fluoresceína.

Em uma série clínica, Ouriel et al. ${ }^{62}$ estudaram o uso do presente método para atestar a viabilidade intestinal de pacientes que passaram por reconstrução de aorta abdominal e verificaram concordância entre os resultados obtidos com o método e a avaliação colonoscópica pósoperatória desses pacientes.

Outros estudos experimentais ${ }^{2,4,94}$ demonstraram a ineficácia da fotopletismografia na diferenciação entre os variados graus de isquemia do intestino.

Os resultados obtidos com a fotopletismografia apontam para a necessidade de um refinamento da técnica, com a tentativa de se quantificar os gráficos fotopletismográficos, visando assim a obtenção de uma relação mais fidedigna entre a taxa de reflecção e o volume sanguíneo tecidual ${ }^{39,71}$, podendo, portanto, se quantificar melhor grau de isquemia intestinal. 


\section{RELAPAROTOMIA}

Muitas vezes os métodos de avaliação da viabilidade intestinal não conseguem por si só precisar a vitalidade do intestino, sendo nesses casos indicada a relaparotomia para uma nova avaliação dos segmentos duvidosos remanescentes na cavidade $^{70}$. Essa relaparotomia é especialmente importante nos casos em que segmentos extensos do intestino se apresentem duvidosos ou nos casos que apresentem infarto mesentérico hemorrágico (devido à recidivas de trombose e possíval propagação de coágulos residuais ${ }^{71}$ ).

A relaparotomia deve ser realizada nas primeiras 18 a 48 horas de pós-operatório, podendo ser efetuada mais precocemente na presença de um quadro de deterioração do paciente ${ }^{70}$. Segundo os autores favoráveis à essa conduta ${ }^{70,78}$, uma vez julgada necessária (na primeira intervenção cirúrgica), a relaparotomia deve ser efetuada independentemente de uma boa evolução pós-operatória do quadro clínico do paciente.

Apesar das desvantagens de se submeter o paciente a uma reintervenção precoce, a relaparotomia é capaz detectar precocemente casos que estejam evoluindo desfavoravelmente, possibilitando sua correção antes que o quadro clínico do paciente se deteriore ${ }^{71}$.

Muitos autores discordam da decisão de se fazer a relaparotomia, uma vez que em cerca de $35 \%$ dessas intervenções as alças se encontram viáveis e em boas condições ${ }^{49,54,87,88}$. Estes autores recomendam a relaparotomia apenas em pacientes que evoluam com disfunção orgânica progressiva, bacteremia e sinais peritoneais, adotando essa conduta com o objetivo de evitar a exposição dos pacientes a riscos desnecessários secundários a laparotomia. Sabe-se, no entanto, que as intervenções realizadas após o aparecimento desses sinais são sempre de alto risco e não revertem a disfunção orgânica do paciente, mesmo após a ressecção do intestino inviável e/ou a drenagem do foco séptico ${ }^{70}$.

No o intuito de propor uma solução para essa indecisão, muitos autores ${ }^{36,54,59,69,84,87,88}$ lançaram mão do uso da laparoscopia para a reavaliação da cavidade abdominal. O uso da laparoscopia ao invés da laparotomia implica em menor morbidade para os pacientes que não evoluem com deterioração das alças intestinais duvidosas e que portanto não necessitariam de nova intervenção cirúrgica $^{54}$.

Para a realização dessa técnica, um trocarte é deixado na cavidade abdominal do doente (de preferência na fossa ilíaca esquerda ${ }^{88}$ ) no ato da primeira cirurgia, sendo feitas vídeo-laparoscopias periódicas para avaliação da vitalidade intestinal. A colocação de uma sonda de Foley com o balão inflado na extremidade do trocarte (visando a proteção das alças intestinais) e o uso de óxido nitroso (pois provoca menos irritação peritoneal) ao invés de $\mathrm{CO}_{2}$ para a realização do pneumoperitônio são fatores que aumentam a segurança do método ${ }^{70}$.

\section{DISCUSSÃO}

O método ideal para a avaliação da viabilidade intestinal intra-operatória deve preencher alguns prérequisitos principais como: 1) poder estar prontamente disponível em qualquer centro cirúrgico que lide com emergências abdominais; 2) ser de fácil aplicação, não necessitando pessoal especializado para a operação do equipamento; 3) ser eficaz e exato, apresentando o mínimo possível de falso-negativos, e principalmente de falsopositivos (uma vez que a permanência de intestino inviável na cavidade pode implicar em consequências nefastas para o paciente); 4) possuir técnica objetiva e de fácil reprodução e 5) apresentar custos compensativos ${ }^{39}$. A Tabela 1 apresenta um resumo da aplicabilidade de cada método analisado na presente revisão.

Tabela 1. Resumo da aplicabilidade dos métodos para avaliação da viabilidade intestinal perioperatória

\begin{tabular}{lll}
\hline Método & Custo & Acurácia \\
\hline Eletromiografia & ++ & ++ \\
Oximetria de superfície & ++ & ++ \\
Oximetria de pulso & +++ & +++ \\
Laser Doppler sem contato & ++++ & ++++ \\
Imagem térmica & ++++ & ++ \\
Critérios clínicos & - & + \\
Doppler ultrassom & + & +++ \\
Laser Doppler & +++ & ++++ \\
Uso da Fluoresceína & + & +++ \\
Termometria & + & ++ \\
pH e PCO & ++ & + \\
Fotopletismografia & +++ & +
\end{tabular}

A eletromiografia, após a recente instituição de melhorias tecnológicas tanto na técnica quanto nos equipamentos, mostrou a necessidade de seu estudo em séries clínicas para uma melhor avaliação de sua acurácia nos seres humanos. Sendo esta provada, e com a dissolução de problemas técnicos e a diminuição dos seus custos, a eletromiografia talvez possa se tornar um método de factível aplicabilidade.

Apesar do DU, devido à sua simplicidade e disponibilidade na sala de cirurgia, ser o método mais aceito e utilizado em geral, alguns estudos relatam resultados inconsistentes relativos a incidência tanto de falso-negativos quanto de falso-positivos, além da sua baixa eficácia em isquemias por obstrução venosa e em estrangulamentos de alça por mais de 2 horas apontando, portanto, para a necessidade de cautela com seu manejo no intra-operatório. O Doppler Ultrasom mostra a apenas necrose nas oclusões venosas. A ausência de pulso evidencia necrose mas a presença a presença não confirma viabilidade. Segundo Savassi-Rocha ${ }^{13}$ é bom na determinação dos pontos ótimos de ressecção. Horgan e Gorey $^{71}$ sugerem que seria interessante combinar o DU 
com a fluoresceinoscopia. O laser-doppler apresenta-se como um método melhor em relação ao DU, mas a difusão do mesmo parece estar ancorada devido ao seu alto custo. O laser-Doppler sem contato e a Imagem térmica mostramse como métodos vanguardistas, cuja aplicabilidade se limita tanto pela escassez de estudos a seu respeito quanto por seu alto custo. Os resultados com a Imagem térmica apontam para uma alta taxa de resultados falso-negativos, o que não se observa no laser-doppler sem contato.

A injeção de fluoresceinoscopia aparece como um método de semelhante aceitação à do Doppler ultrassom. Este, apesar de parear com o Doppler nos custos, apresenta menor praticidade e sua acurácia varia entre os autores, havendo aqueles que o consideram mais ou menos efetivo em relação ao Doppler. A inserção do fluorômetro aumenta sobremaneira a acurácia do método, apesar de incutir em maiores custos e maior demanda tecnológica.

A termometria mostra-se um método de aplicação rápida e baixos custos, porém faz-se necessário um maior número de estudos para a avaliação da incidência de resultados falso-positivos e falso-negativos, uma vez que está provado que o desaparecimento do gradiente térmico não significa necessariamente necrose ${ }^{12}$. Com relação a pHmetria e a tonometria, existem, segundo autores ${ }^{39,71}$, limitações com o uso do método devido à sua ineficácia na avaliação da viabilidade em situações de isquemia mais tardia ou devido ao não-estabelecimento de valores críticos de $\mathrm{pH}$ e $\mathrm{PCO}_{2}$ que se correlacionem com a isquemia tecidual. Dessa forma, os resultados e custos desse método lhe atribuem importância secundária frente aos outros métodos existentes, não tendo, portanto, muita chance de se tornar um método difundido.

Apesar do bom resultado do estudo de Sheridan et al., muitos estudos ainda devem ser conduzidos visando a avaliação da acurácia da oximetria de superfície em pacientes. Com relação à oximetria de pulso, já foi relatado $^{53}$ que esse método pode apresentar o mesmos resultados da oximetria de superfície, mas a sua praticidade e menor tempo de aplicação lhe dão a preferência. A oximetria de pulso apresenta, porém custos mais altos. Como já foi dito, a necessidade do refinamento da técnica e de seu estudo é importante para a aplicabilidade da fotopletismografia, isso aliado à diminuição de seus custos.

Deve sempre ser considerada a possibilidade da realização das relaparotomias em casos que mesmo após a utilização de algum dos métodos descritos se mostrem duvidosos. O uso da laparoscopia nesses casos é interessante e muito factível, já que diminue a morbidade dos casos evoluiriam bem.

\section{CONCLUSÃO}

Pudemos observar que, apesar da extensa literatura existente a respeito do assunto, existem poucos estudos amplos e revisões de literatura que comparem satisfatoriamente os métodos entre si. Além disso, as metodologias utilizadas nos estudos experimentais existentes na maioria das vezes são pouco comparáveis entre si diferindo em fatores como tempo de isquemia, padrão de isquemia, modelo animal (apesar do canino predominar), etc. Dessa forma, podemos concluir que os métodos disponíveis não apresentam estudos que se comparem suficientemente para mostrar logisticamente a aplicabilidade segura e a unanimidade na escolha do melhor deles.

Apesar disso, é factível que os métodos auxiliares mais utilizados, devido ao seu melhor custo-benefício, são o Doppler ultrassom e a injeção intravenosa de fluoresceína, devendo-se considerar também como um procedimento seguro e de bons resultados a utilização da monitoração laparoscópica pós-operatória, principalmente nos casos em que a avaliação da viabilidade intestinal no intra-operatório não foi satisfatória.

Esteves FP, Caravatto PPP, Yamakami LYS, Araújo SEA, Genzini T, Perosa M. Perioperative assessment of the intestinal viability. Rev Med (São Paulo) 2002 jan.-dez.;82(1-4):46-57.

\begin{abstract}
The assessment of a intestinal segment's viability and its degree of ischemia only by clinical criteria is often hard for the surgeon. Sometimes it's necessary to use auxiliary methods to assess the degree of intestinal injury, which sometimes is imperceptible by clinical evaluation. This review presents the principal methods to undergo this evaluation, describing their technique and necessary equipament, as long as their results. The analized methods were divided in experimental: eletromiography, non-contact tissue laser Doppler and thermal imaging; and clinical: clinical criteria, Doppler ultrasonography, laser Doppler, intravascular dyes, surface oximetry, pulse oxymetry, thermography, $\mathrm{pH}$ measurement, tonometry and infrared photopletysmography. The results of second-look laparotomies in patients with mesenteric vascular insufficiency who were operated are described too. After reviewing all the literature, we found that Doppler ultrasound and fluorescein injection are, due to its costs and easy menagement, the most used and accepted methods to assess the perioperative intestinal viability. We had also observed that post-operative laparoscopy has a high value on the monitorization of the intestinal viability in patients that undergo surgical treatment for mesenteric vascular insufficiency, especially if the introperative assessment of their intestinal viability wasn't do precise.
\end{abstract}

KEYWORDS: Review litterature. Perioperative care. Digestive system surgical. Procedures methods. Ischemia/complications. Intestines/pathology. Intestines/blood suply. Splanchmic circulation. 


\section{REFERÊNCIAS}

1. Ahn H, Lindhagen J, Nilsson GE, Salerud EG, Jodal M, Lundgren O. Evaluation of laser Doppler flowmetry in the assessment of intestinal blood flow in the cat. Gastrenterology 1985;88:951-7.

2. Alos R, Garcia-Granero E, Calvete J, Uribe N. The use of Photoplethysmography and Doppler ultrasound to predict anastomotic viability after segmental intestinal ischemia in dogs. Eur J Surg 1993;159:35-41.

3. Ando M, Ito M, Nihei Z, Sugihara K. Assessment of intestinal viability using a non-contact laser tissue blood flowmeter. Am J Surg 2000;180:176-80.

4. Avino AJ, Oldenburg WA, Gloviczki P, Miller VM, Burgart LJ, Atkinson EJ. Inferior mesenteric venous sampling to detect colonic ischemia: a comparison with laser Doppler flowmetry and photoplesthymography. J Vasc Surg 1995;22:271-7.

5. Basdanis G, Zisiadis A, Michalopoulos A, Papadopoulos V, Apostolidis S, Katsohis C. Myoeletric assessment of large bowel viability: na experiment in dogs. Eur J Surg 1999;165:1182-6.

6. Bergman RT, Gloviczki P, Welch TJ, Naessens JM, Bower TC, Hallett JW Jr, et al. The role of intravenous fluorescein in the detection of colon ischemia during aortic reconstruction. Ann Vasc Surg 1992;6:74-9.

7. Bjorck M, Lindberg F, Broman G, Bergqvist D. pHi monitoring of the sigmoid colon after aortoiliac surgery. A five-year prospective study. Eur J Endovasc Surg 2000;20:273-80.

8. Boyle NH, Manifold D, Jordan MH, Mason RC. Intraoperative assessment of colonic perfusion using scanning laser Doppler flowmetry during colonic resection. J Am Coll Surg 2000;191:504-10.

9. Brolin RE, Orland PJ, Bibbo C, Reddell MT, Fedorciw B, Gazi G, et al. Comparison of blood flow and myoeletric measurements in two chronic models of mesenteric ligation. Arch Surg 1995;130:147-52.

10. Boley SJ, Sprayregan S, Siegelman SS, Veith FJ. Initial results of an aggressive roentgenological and surgical approach to acute mesenteric ischemia. Surgery 1977;82:848-55.

11. Brolin RE, Semmlow JL, Koch RA, Reddell MT, Mast BA, Mackenzie JW. Myoelectric assessment of bowel viability. Surgery 1987;102:32-8.

12. Brolin RE, Semmlow JL, Mackenzie JW, et al. Quantitative evaluation of bowel viability. Porc IEEE Front Eng Comput Health Care 1984;6:320.

13. Brolin RE, Semmlow JL, Mackenzie JW, Reddell MT. Quantitative myoeletric determination of bowel viability. J Surg Res 1986;41:557-62.

14. Brolin RE, Semmlow JL, Sehonanda A, Koch RA, Reddell MT, Mast BA, et al. Comparison of five methods of assessment of intestinal viability. Surg Gynecol Obstet 1989;168:6-12.

15. Brooks JP, Perry WB, Putnam AT, Karulf RE. Thermal imaging in the detection of bowel ischemia. Dis Colon Rectum 2000;43:1319-21.

16. Bulkley GB, Zuidema GD, Hamilton SR, O’Mara CS, Klacsmann PG, Horn SD. Intraoperative determination of viability following ischemic injury. Ann Surg 1981;193:628-37.

17. Cabot RM, Kohatsu S. The effects of ischemia on the electrical and contractile activities of the canine small intestine. Am J Surg 1984;136,242-6.

18. Cappel MS. Intestinal vasculopathy I. Gastroenterol Clin North Am 1998;27:783-825.

19. Carter K Jr, Cherry G, Myers MB. Determination of the viability of ischemic intestine. Arch Surg 1970;100:695701.

20. Carter MS, Fantini GA, Sammartano RJ, Mitsudo S, Silverman DG, Boley SJ. Qualitative and quantitative fluorescence for determining intestinal viability. Am J Surg 1984;147:117-23.

21. Chou CC. Relationship between intestinal blood flow and motility. Annu Rev Physiol 1982;44:29-42.

22. Cooperman M, Martin EW Jr, Carey LC. Determination of intestinal viability by Doppler ultrasonography in venous infarction. Ann Surg 1980;191:57-8.

23. Cooperman M, Martin EW Jr, Carey LC. Evaluation of ischemic intestine by Doppler ultrasound. Am J Surg 1980;139:73-7.

24. Cooperman M, Pace WG, Martin EW Jr, Pflug B, Keith LM Jr, Evans WE, et al. Determination of viability of ischemic intestine by Doppler ultrasound. Surgery 1978;83:705-10.

25. DeNobile J, Guzzetta P, Patterson K. Pulse oximetry as a means of assessing bowel viability. J Surg Res 1990;48:213.

26. Dineen P, Goulian D, McSherry CK. A method of demonstrating intestinal viability. Am J Gastroenterol 1966;45:335-41.

27. Diniz. Determinação do tempo de desaparecimento dos sinais Doppler e do gradiente térmico na isquemia intestinal de origem venosa e sua correlação com a viabilidade do intestino. Estudo experimental em cães [Tese]. Belo Horizonte: Faculdade de Medicina, Universidade Federal de Minas Gerais; 1992.

28. DiResta GR, Corbally MT, Sigurdson ER, Haumschild D, Ridge R, Brennan MF. Infrared laser Doppler flowmeter in the determination of small bowel perfusion after ischemic injury: comparison with the clearence of locally generated hydrogen and fluorescein angiography. J Pediatr Surg 1994;29:1352-5.

29. Enquist IF, Kremen AJ. Determination of intestinal viability; an experimental evaluation of multiple tests. Surg Forum 1952-1953;98(38th Congress):87-94.

30. Eypasch E, Troidl H, Mennigen R, Spangenberger W, Barlow AP. Laparoscopy via an indwelling cannula: an alternative to planned relaparotomy. $\mathrm{Br} \mathrm{J}$ Surg 1992;79:1368.

31. Feld AD, Fondacaro JD, Holloway GA Jr, Jacobson ED. Measurement of mucosal blood flow in the canine intestine with laser Doppler velocimetry. Life Sci 1982;31:1509-17.

32. Galandiuk S, Fazio VW, Petras RE. Fluorescein endoscopy. A technique for noninvasive assessment of intestinal ischemia. Dis Col Rectum 1988;31:848-53.

33. Gorey TF. Prediction of intestinal recovery after ischemic injury due to arterial, venous and mixed occlusions. J R Soc Med 1980;73:631-4. 
34. Guisan YJ, Hreno A, Gurd FN. Effect of acute ischemia in the motility of the awake dog. Eur Surg Res 1975;7:23-33.

35. Guslandi M, Sorghi S, Polli D, Tittobello A. Measurement of rectal blood flow by laser Doppler flowmetry in inflammatory bowel disease. Hepatogastrenterology 1998;45:445-6.

36. Hamzaoglu I, Saribeyoglu K, Karahasanoglu T, Apaydin B, Bayrak I, Sirin F, et al. Can laparoscopy be performed safely earlier after laparotomy? Surg Laparosc Endosc Percutan Tech 2000;10:379-81.

37. Herrlin JO, Glasser ST, Lange K. New methods for determining the viability of the bowel. Arch Surg 1942;45:785.

38. Hobson RW 2nd, Wright CB, O'Donnell JA, Jamil Z, Lamberth WC, Najem Z. Determination of intestinal viability by Doppler ultrasound. Arch Surg 1979;114:165-8.

39. Horgan PG, Gorey TF. Operative assessment of intestinal viability. Surg Clin North Am 1992;1:141-53.

40. Johansson K, Ahn H, Lindhagen J. Assessment of small-bowel ischemia by laser Doppler flowmetry. Some case reports. Scand J Gastrenterol 1986;21:1147-52.

41. Johansson K, Ahn H, Lindhagen J. Intraoperative assessment of blood flow and tissue viability in small-bowel ischemia by laser Doppler flowmetry. Acta Chir Scand 1989;155:341-6.

42. Kam DM, Scheeres DE. Fluorescein-assisted laparoscopy in the identification of arterial mesenteric ischemia. Surg Endosc 1993;7:75-8.

43. Katz S, Wahab A, Murray W, Williams LF. New parameters of viability in ischemic bowel disease. Am J Surg 1974; 127:136-41

44. Khin J, Daniel EE. The effects of ischemia on intestinal nerves and electrical slow waves. Am J Dig Dis 1970;15:959-81.

45. Knichwitz G, Brussel T, Reinhold P, Schaumann F, Richter $\mathrm{KD}$, Van Aken $\mathrm{H}$. Early onset of regional intestinal ischemia can be detected with carbon dioxide tension inside the peritoneal cavity. Anesth Analg 2000;91:1182-7.

46. Kwong KW, Fraser RE, Paton BC. Assessment of vascular integrity of intestinal segments by dye injection. Br J Surg 1976;54:144-6.

47. Lammers WJ, el-Kays A, Manefield GW, Arafat K, elSharkawy TY. Disturbances in the propagation of the slow wave during acute local ischemia in the feline small intestine. Eur J Gastroenterol Hepatol 1997;9:381-8.

48. Lanzafame RJ, Naim JO, Francis M, Tomkiewicz ZM, Voytek A, Hinshaw JR. Prediction of blood flow in and viability of ischemic small bowel: fluorescein perfusion versus radiolabeled microspheres. Curr Surg 1983;40:286-9.

49. Leveg PJ, Krausz MM, Manny J. The role of second-look procedure in improving survival time for patients with mesenteric venous trombosis. Surgery 1990;170:287-91.

50. Liu DL, Svanberg K, Wang I, Andersson-Engels S, Svanberg S. Laser Doppler perfusion imaging: new technique for determination of perfusion and reperfusion of splanchnic organs and tumor tissue. Lasers Surg Med 1997;20:473-9.

51. Locke R, Hauser CJ, Shoemaker WC. The use of surface oximetry to assess bowel viability. Arch Surg 1984;119:12526.

52. Lynch TG, Hobson RW 2nd, Kerr JC, Brousseau DA, Silverman DG, Reilly CA, et al. Doppler ultrasound, laser Doppler, and perfusion fluorometry in bowel ischemia. Arch Surg 1988;123:483-6.
53. MacDonald PH, Dinda PK, Beck IT, Mercer CD. The use of oxymetry in determing intestinal blood flow. Surg Gynecol Obstet 1993;176:451-8.

54. MacSweeney STR, Postlethwaite JC. "Second-look" laparoscopy in the management of acute mesenteric ischemia. Br J Surg 1994;81:90.

55. Mann A, Fazio VM, Lucas FV. A comparative study of the use of fluorescein and Doppler device in the determination of intestinal viability. Surg Gynecol Obstet 1982;145:53-5.

56. Matern U, Haberstroh J, el Saman A, Pauly E, Salm R, Farthmann EH. Emergency laparoscopy. Technical support for the laparoscopic diagnosis of intestinal ischemia. Surg Endosc 1996;10:883-7.

57. Myers MB, Cherry G, Gesser J. Relationship between surface $\mathrm{pH}$ and $\mathrm{pCO}_{2}$ and the vascularity and viability of the intestine. Surg Gynecol Obstet 1972;134:787-9.

58. Myers MB, Cherry G. Use of vital dyes in the evaluation of the blood supply of the colon. Surg Gynecol Obstet 1969;128:97-102.

59. Nassar AH, Htwe T, Hefny H, Kholeif Y. The abdominal drain. A convenient port for second-look laparoscopy. Surg Endosc 1996;10:1114-5.

60. O'Donnell JA, Hobson RW. Operative confirmation of Doppler ultrasound in evaluation of intestinal viability. Surgery 1980;87:109-12.

61. Orland PJ, Cazi GA, Semmlow JL, Reddell MT, Brolin RE. Determination of small bowel viability using quantitative myoeletric and color analysis. J Surg Res 1993;55:581-7.

62. Ouriel K, Fiore WM, Geary JE. Detection of occult colonic ischemia during aortic procedures: use of an intraoperative photoplethysmography technique. J Vasc Surg 1988;7:5-9.

63. Paes E, Vollmar JF, Hutschenreiter S, Schoenberg MH, Kubel R, Scholzel E. Mesenteric infarct: new aspects of diagnosis and theraphy. Chirurg 1988,59:828-35.

64. Papachristou D, Fortner JG. Prediction of intestinal by intraarterial dye injection: a simple test. Am J Surg 1976;132:572-

65. Pearce WH, Jones DN, Warren GH, Bartle EJ, Whitehill TA, Rutherford RB. The use of infrared photoplethismography in identifying early intestinal ischemia. Arch Surg 1987;122:30810.

66. Redaelli CA, Schiling MK, Buchler MW. Intraoperative laser Doppler flowmetry: a predictor of ischemic injury in acute mesenteric infarction. Dig Surg 1998;15:55-9.

67. Redaelli CA, Schiling MK, Carrel TP. Intraoperative assessment of intestinal viability by laser Doppler flowmetry for surgery of rupted abdominal aortic aneurysms. World J Surg 1998;22:283-9.

68. Rotering RH Jr, Dixon JA, Holloway GA Jr, McCloskey DW. A comparison of the He Nelaser and ultrasound Doppler systems in the determination of viability of isquemic canine intestine. Ann Surg 1982;196:705-8.

69. Sackier J. Diagnostic laparoscopy in non-malignant disease. Surg Clin North Am 1992;72:1033-43.

70. Savassi Rocha PR. Avaliação peroperatória da viabilidade intestinal. In: Rasslan S. O doente cirúrgico na UTI. CBMI Série Clínicas Brasileiras de Medicina Intensiva. Ano 6 Volume 10 2001. p.412-29.

71. Savassi-Rocha PR. Métodos de avaliação da viabilidade intestinal. In: Rasslan S. Afecções cirúrgicas de urgência. São Paulo: Robe; 1995. Cap. 15, p.186. 
72. Savassi-Rocha PR, Rausch M. Temperature difference between mesenteric and antimesenteric intestinal margins in men as a criterious of intestinal viability. Dig Dis Sci 1986;31:317.

73. Savassi-Rocha PR, Diniz MTC. Determination of time disappearence of Doppler signals and of thermal gradient in intestinal ischemia of venous origin and its correlation with intestinal viability. ABCD Arq Bras Cir Dig 1995;10:78.

74. Savassi-Rocha PR, Tobon MJC, Rodrigues MAG, et al. Determination of the optimum points of resection in desvacularized intestinal loops by intravenous injection of patent V blue. Braz Arch Dig Surg 1991;6:101-5.

75. Savassi-Rocha PR. Determinação dos pontos ótimos de ressecção de alças intestinais iequêmicas pelo doplerometria, termometria e fluoresceinoscopia. Estudo comparativo experimental em cães [Tese]. Belo Horizonte: Faculdade de Medicina, Universidade Feceral de Minas Gerais; 1983.

76. Semmlow JL, Orland PJ, Reddell MT, Brolin RE. Evaluation of quantitative aproaches to assessment of bowel viability. Biomed Instrum Technol 1997;31:591-9.

77. Shah SD, Andersen CA. Prediction of small bowel viability using doppler ultrasound: clinical and experimental evaluation. Ann Surg 1981;194:97-9.

78. Shaw RD. Superior mesenteric embolectomy in the treatment of massive mesenteric infarction. N Eng Med J 1957;257:595.

79. Shepherd AP, Riedel GL. Continuous measurement of mucosal blood flow by laser Doppler velocimetry. Am J Physiol 1982;242:G668-72.

80. Sheridan WG, Lowndes RH,Willians GT, Young HL. Determination of a critical level of tissue oxygenation in acute intestinal ischemia. Gut 1992;33:762-6.

81. Silverman DG, Cedrone FA, Hurford WE, Bering TG, LaRossa DD. Monitoring tissue elimination of fluorescein with the perfusion fluorometer: a new method to assess capillary blood flow. Surgery 1981;90:409-17.

82. Silverman DG, LaRossa DD, Barlow CH, Bering TG, Popky LM, Smith TC. Quantification of tissue fluorescein delivery and prediction of flaps viability with the fiberoptic dermofluorometer. Plast Reconstruct Surg 1980;66:545-53.

83. Silverman DG, Hurford WE, Cooper HS, Robinson M, Brousseau DA. Quantification of fluorescein distribution to strangulated rat ileus. J Surg Res 1983;34:179-86.

84. Slutzki S, Halpern Z, Negri M, Kais H, Halevy A. The laparoscopy second-look for ischemic bowel disease. Surg Endosc 1996;10:729-31.

85. Stein MR, Parker CW. Reactions following intravenous fluorescein. Am J Ophthalmol 1971,72:861-8.

86. Stolar CJH, Randolf JG. Evaluation of ischemic bowel viability with a fluorescent technique. J Pediatr Surg 1978;13:221-5.

87. Tiberio G. Líschemia intestinale acuta. Atti della Societè Italiana di Chirurgia, 1: 312, 1992.

88. Tola N, Portoguese A, Maniga AM. Il "second-look" laparoscópico nell'a ischemia intestinale acuta. Minerva Chirur 1997;52:527.

89. Turkyilmaz Z, Sonmez K, Basaklar AC, Demirogullari B, Numanoglu V, Ekingen G, et al. Assessment of anastomotic reliability with pulse oximetry in graded intestinal ischemia: an experimental study in dogs. J Pediatr Surg 1997;32: 172831.

90. Vahl AC, Ozkaynak-Yilmaz EN, Nauta SH, Scheffer GJ, FeltBersma RJ, Brom HL, et al. Endoluminal pulse oximetry combined with tonometry to monitor the perfusion of the sigmoid during and after resection of abdominal aortic aneurysm. Cardiovasc Surg 1997;5:65-70.

91. Verastegui TCV. Determinação da viabilidade intestinal de alças estranguladas e revascularizadas por critérios clínicos, doplerometria e termometria. Estudo experimental em cães [dissertação]. Belo Horizonte: Faculdade de Medicina, Universidade Federal de Minas Gerais; 1988.

92. Wapnick S, Solowiejczyk M, Grosman E. Detection of intestinal ischemia: part 2. Temperature difference between the mesenteric and antimesenteric margin of bowel as criterious of intestinal anastomosis viability. Rev Surg 1977;34:371-5.

93. Wapnick S, Solowiejczyk M, Shiloh R. Detection of intestinal ischemia: part 1: microradiological and temperature differences between mesenteric and antimesenteric margin of small intestine of the rat. J Surg Res 1976,21:403-7.

94. Whitehill TA, Pearce WH, Rosales C, Yano T, Van Way CW, Rutherford RB. Detection thresholds of nonocclusive intestinal hypoperfusion by Doppler ultrasound, photoplesthymography, and fluorescein. J Vasc Surg 1988;8:28-32

95. Wright CB, Hobson RW. Prediction of intestinal viability using Doppler ultrasound technics. Am J Surg 1975;129:642-5. 